ISSN 1991-8631

Original Paper

http://indexmedicus.afro.who.int

\title{
Géochimie des laves basaltiques récentes des zones Nord et Est de Ngaoundéré (Cameroun, Plateau de l'Adamaoua, Afrique centrale): pétrogenèse et nature de la source
}

\author{
Oumarou Faarouk NKOUANDOU ${ }^{1 *}$, Ismaïla NGOUNOUNO ${ }^{2}$ et Bernard DERUELLE ${ }^{3}$ \\ ${ }^{1,2}$ Département des Sciences de la Terre, Faculté des Sciences, Université de Ngaoundéré, B.P. 454 \\ Ngaoundéré, Cameroun.E-mail: ofaarouk@yahoo.fr; ngounouno@yahoo.fr \\ ${ }^{3}$ Laboratoire de Magmatologie et Géochimie Inorganique et Expérimentale, Institue de Physique du Globe de \\ Paris, UMR 7154, Université Pierre-et-Marie-Curie et IUFM Académie de Versailles, 4, place Jussieu, 75252 \\ Paris cedex05, France.E-mail: déruelle@ccr.jussieu.fr; déruelle@yahoo.fr \\ *Auteur correspondant, E-mail : ofaarouk@yahoo.fr
}

\section{RESUME}

Des coulées et projections basaltiques récentes (basaltes: I.D. $<35$, hawaiites: $35<$ D.I. $<50$ et mugéarites: 50 < D.I. < 65) contenant des cristaux d'olivine, de clinopyroxène, d'oxydes de fer-titane, de plagioclase, de kaersutite, de feldspaths alcalins, d'apatite et des xénocristaux de feldspath (basaltes), affleurent au Nord et à l'Est de Ngaoundéré (Plateau de l'Adamaoua). Elles se sont cristallisées entre 1170 et $1300{ }^{\circ} \mathrm{C}( \pm$ $100^{\circ} \mathrm{C}$ ). La différenciation des laves est dominée par la cristallisation fractionnée. Les basaltes de Ngaoundéré proviendraient d'un faible taux de fusion partielle d'une source mantellique de type FOZO.

(C) 2010 International Formulae Group. All rights reserved.

Mots clés : Pétrologie, géochimie, laves basaltiques récentes, FOZO, Plateau de l’Adamaoua, Cameroun.

\section{INTRODUCTION}

Le Plateau de l'Adamaoua (Figure 1) est une structure volcano-tectonique en horst limitée au Nord par les failles de l'Adamaoua et au Sud par les failles de Djérem et de Mbéré (Dumont, 1987). Lasserre (1961) a regroupé les formations volcaniques (Figure 2) sur le Plateau de l'Adamaoua en trois séries d'émissions par rapport aux observations de Gèze (1943) dans l'Ouest du Cameroun: (1) la série basaltique ancienne d'âge Crétacé terminal (Lasserre, 1961), (2) la série intermédiaire à dominance trachytique et phonolitique et (3) la série basaltique récente. La série ancienne, fortement latéritisée, représente les $3 / 5$ de la surface couverte par les formations volcaniques. Elle serait de type fissural et ne présente plus d'appareils volcaniques visibles. La série intermédiaire à dominance trachytique et phonolitique d'âge Miocène (Nkouandou et al., 2008) est représentée par 35 necks, dômes et dômescoulées. La série basaltique récente d'âge MioPliocène $(10,0$ à 7,0 $\pm 0,2$ Ma et $11,39 \pm 0,03$ à 9,28 $\pm 0,03 \mathrm{Ma}$ ) (Gouhier et Nougier, 1974; Marzoli et al., 1999 ; Nkouandou et al., 2008) est représentée par trois types de coulées basaltiques dont (1) les coulées inférieures et (2) supérieures essentiellement constituées de basalte (avec les ankaramites pour les coulées supérieures), affleurent respectivement dans les bas-fonds et aux sommets des collines et, 
(3) les coulées intermédiaires constituées de basalte, hawaiite et mugéarite affleurent sur les pénéplaines et les flancs des collines. Le volcanisme du Plateau de l'Adamaoua est très peu étudié par rapport au reste de la 'Ligne du Cameroun'. Les volcans des zones au Nord et à l'Est de Ngaoundéré (Figure 2) offrent l'opportunité d'étudier la pétrogenèse des laves basaltiques en domaine continental.

Les objectifs de cet article sont d'utiliser les outils de la pétrographie et de la géochimie élémentaire (éléments majeurs et traces) et isotopiques $(\mathrm{Sr}, \mathrm{Nd}$ et $\mathrm{Pb})$ pour identifier les processus magmatiques et de déterminer la nature de la source du magmatisme récent des zones Nord et Est de Ngaoundéré.

\section{Cadre géologique}

Le socle de l'Adamaoua est constitué de roches métamorphiques et de granitoïdes liés à l'orogenèse panafricaine $(615 \pm 27$ à $652 \pm 10 \mathrm{Ma})$ ou antérieurs $(880 \pm 55$ à 1008 $\pm 65 \mathrm{Ma}$ ) (Tchameni et al., 2006). Il est intensément découpé par un faisceau d'accidents majeurs (Moreau et al., 1987). Une distension subméridienne au Cénozoïque est à l'origine de la surrection du horst de l'Adamaoua et de l'effondrement du fossé de Djérem et de Mbéré (Ngangom, 1983), surrection qui a engendré une large anomalie de Bouger (-80 à $-100 \mathrm{mgl}$ et $-120 \mathrm{mgl} / \mathrm{cm}$ ) (Brown et Fairhead, 1983; Poudjom Djomani, 1995 ; Okereke, 1988). La croûte continentale sous le plateau est mince $(23 \mathrm{~km}$, Poudjom Djomani, 1995) au nord et surmonte un manteau anormalement chaud (Dorbath et al., 1986) caractérisé par une faible vitesse de propagation des ondes "P" $(7,8 \mathrm{~km} / \mathrm{s})$ par rapport au sud où elle est normale $(33 \mathrm{~km}$ et $8,0 \mathrm{~km} / \mathrm{s}$ ) (Dorbath et al., 1984; Stuart et al., 1985). La mise en place du volcanisme est vraisemblablement liée à des fractures panafricaines (Cornacchia et Dars, 1983; Moreau et al., 1987). Au sud de Ngaoundéré, des cônes de pyroclastites sont présents avec parfois des lacs de cratère.

\section{Conditions analytiques}

Les conditions analytiques de la chimie des minéraux sont les suivantes: olivine et clinopyroxène: $15 \mathrm{kV}$ et $40 \mathrm{nA}, 20 \mathrm{~s}$ par élément sauf Si pour l'olivine (10 s) et Ti pour le clinopyroxène $(30 \mathrm{~s})$; plagioclase, Kfeldspath: $15 \mathrm{kV}, 10 \mathrm{nA}$ et $10 \mathrm{~s}$ par élément; titanomagnétite: $20 \mathrm{kV}$ et $40 \mathrm{nA}$ par élément ; $10 \mathrm{~s}$ pour $\mathrm{Si}, \mathrm{Ca}$ et $\mathrm{Ni} ; 25 \mathrm{~s}$ pour $\mathrm{Mn} ; 15 \mathrm{~s}$ pour $\mathrm{Cr}$; $30 \mathrm{~s}$ pour $\mathrm{Al}$; $40 \mathrm{~s}$ pour $\mathrm{Ti}, \mathrm{Fe}(\mathrm{F} 3+$ recalculé d'après Stormer, 1983) et $\mathrm{Mg}$; kaersutite: $15 \mathrm{kV}$ et $21 \mathrm{nA}$ pour tous les éléments ; $15 \mathrm{~s}$ pour $\mathrm{Si}, \mathrm{Al}, \mathrm{Mg}, \mathrm{Na}, \mathrm{K}$, et $\mathrm{Ba}$; $20 \mathrm{~s}$ pour $\mathrm{Ti}$ et $\mathrm{Ca}, 25 \mathrm{~s}$ pour $\mathrm{Fe}$ et $\mathrm{Mn}, 30 \mathrm{~s}$ pour $\mathrm{F}$ et $\mathrm{Cl}(\mathrm{Fe} 3+$ recalculé selon Leake et al., 1997). Le programme de correction «PAP» (Pouchou et Pichoir, 1991). Les formules structurales des minéraux sont calculées sur la base de 4 oxygènes pour l'olivine, 6 oxygènes pour le clinopyroxène, 8 oxygènes pour le plagioclase et feldspath alcalin, 32 oxygènes pour les oxydes (6 oxygènes pour l'ilménite), 23 oxygènes pour l'amphibole et 26 oxygènes pour l'apatite.

Les analyses chimiques des laves représentatives des zones Nord et Est de Ngaoundéré ont été effectuées par ICP-AES et ICP-MS au CRPG, CNRS, Nancy. Les précisions analytiques sont celles décrites par Carignan et al. (2001). Les rapports isotopiques du strontium, du néodyme et du plomb ont été déterminés par spectrométrie de masse (VG Sector 54 TIMS) à l'Université Libre de Bruxelles (ULB).

\section{RESULTATS}

Nomenclature, pétrographie et minéralogie Les laves sont dénommées (Figure 4), d'après leur indice de différenciation, I.D. (Thornton et Tuttle, 1960) : basalte (D.I. < $35)$, hawaiite $(35<$ D.I. < 50) et mugéarite $(50$ $<$ D.I. < 65).

Les basaltes (NG139 et NG130) ont une texture microlitique porphyrique; ils contiennent des phénocristaux (1,5 à $3 \mathrm{~mm})$ d'olivine $\left(\mathrm{Fo}_{78-68}\right), \quad$ de diopside $\left(\mathrm{Wo}_{45-46} \mathrm{En}_{45-49} \mathrm{Fs}_{10-5}\right)$ et d'augite $\left(\mathrm{Wo}_{37-44} \mathrm{En}_{49-50} \mathrm{Fs}_{14-6 n}\right)$ (Figure 3), de titanomagnétite (67\% Usp), de kaersutite ( $\mathrm{Ti}=$ 
0,67 a.p.u.f.; $\mathrm{Mg \#} \mathrm{=61)} \mathrm{et} \mathrm{de} \mathrm{plagioclase}$ (Ab35-47An51-64) dans une matrice constituée de microlites de plagioclase ( $\mathrm{Ab}{ }_{47-58} \mathrm{An}_{41-51}$ ), de diopside et de microcristaux de titanomagnétite $(60 \%$ Usp) et d'ilménite (43,5-39,46\% de $\mathrm{TiO}_{2}$ ). Les températures de cristallisation estimées d'après la calibration de Stormer (1983) sur le couple titanomagnétite - ilménite sont autour de 910 ${ }^{\circ} \mathrm{C} \pm 50{ }^{\circ} \mathrm{C}$ et les fugacités d'oxygène de l'ordre de $10^{-13}$ atm. Le basalte NG130 contient de xénocristaux $(4 \times 3 \mathrm{~mm})$ de sanidine $\left(\mathrm{Or}_{18,4} \mathrm{Ab}_{70,4}\right)$. Des poches de carbonate $(\varnothing=1,5 \mathrm{~cm} ; \mathrm{L}=4 \mathrm{~cm})$ sont présentes dans certains échantillons de basalte.

Les hawaiites (représentées par NG142) ont une texture microlitique porphyrique, avec des microphénocristaux (< 1,5 mm) de plagioclase $\left(\mathrm{An}_{51-54} \mathrm{Ab}_{44-47}\right)$, des phénocristaux de kaersutite $(\mathrm{Ti}=0,680$ a.p.u.f.; $\mathrm{Mg \#} \mathrm{=} \mathrm{58)} \mathrm{très} \mathrm{souvent} \mathrm{déstabilisés} \mathrm{en}$ titanomagnétite (60\% Usp), distribués dans une matrice constituée de microlites d'andésine $\quad\left(\mathrm{An}_{37-49} \mathrm{Ab}_{50-57}\right), \quad \mathrm{de}$ titanomagnétite et de fluoroapatite (jusqu'à 2,3\% de F).

Les mugéarites (NG14, NG8) ont une texture microlitique porphyrique, avec des phénocristaux de diopside $\left(\mathrm{Wo}_{45-47} \mathrm{En}_{39-40} \mathrm{Fs}_{14-15}\right)$, de kaersutite $(\mathrm{Ti}=$ 0,63 a.p.u.f; $\mathrm{Mg \#}=60)$, d'anorthose ( $\left.\mathrm{An}_{9} \mathrm{Ab}_{59} \mathrm{Or}_{32}\right)$, de titanomagnétite (50\% Usp) dans une matrice constituée de microlites d'andésine $\left(\mathrm{An}_{37} \mathrm{Ab}_{57} \mathrm{Or}_{5}\right)$, de microcristaux de titanomagnétite et d'apatite riche en fluor (jusqu'à 2,7\% de F).

\section{Géochimie}

Les analyses chimiques des laves basaltiques récentes des zones Nord et Est de Ngaoundéré sont présentées dans le Tableau 2. Toutes les laves sont à néphéline normative $(1,9<\mathrm{Ne}<13,7)$ avec des teneurs en $\mathrm{SiO}_{2}<$ $50 \%$. Les variations des teneurs en éléments majeurs (Figure 5) et de transition (Figure 6) sont évaluées en fonction des teneurs croissantes de $\mathrm{SiO}_{2}$ et $\mathrm{MgO}$ respectivement. Dans les diagrammes $\mathrm{SiO}_{2}$-majeurs, les teneurs en $\mathrm{TiO}_{2}, \quad \mathrm{Fe}_{2} \mathrm{O}_{3}, \quad \mathrm{MgO}$ et $\mathrm{CaO}$ décroissent et celles en $\mathrm{Al}_{2} \mathrm{O}_{3}, \mathrm{Na}_{2} \mathrm{O}$ et $\mathrm{K}_{2} \mathrm{O}$ croissent des basaltes aux mugéarites. Celles en $\mathrm{P}_{2} \mathrm{O}_{5}$ augmentent pour les basaltes et hawaiites puis diminuent fortement dans les mugéarites. Deux basaltes (NG121 et NG1301) s'écartent des droites de corrélation. Les teneurs en $\mathrm{MgO}$ des laves sont comprises entre 6,7 et $7,7 \%$, mises à part celles élevées de NG110 (10,1\%) qui est une ankaramite.

Dans les diagrammes $\mathrm{MgO}$ - éléments de transition (Figure 6), les teneurs en $\mathrm{V}, \mathrm{Cr}$, $\mathrm{Co}$, $\mathrm{Ni}$ et $\mathrm{Cu}$ décroissent fortement des basaltes aux mugéarites avec les teneurs décroissantes de $\mathrm{MgO}$. Celles en éléments incompatibles (Tableau 2) augmentent des basaltes aux mugéarites. Seul le basalte NG121 a des teneurs relativement faibles de $\mathrm{Zr}$ (213 ppm), Nb (43 ppm), Hf (5 ppm), Ta $(3,21 \mathrm{ppm})$, Th $(3,45 \mathrm{ppm})$ et $\mathrm{U}(0,91 \mathrm{ppm})$. Les teneurs en terres rares du basalte NG121 sont relativement faibles par rapport à celles des autres basaltes.

Les valeurs des teneurs des éléments traces et terres rares des laves basaltiques sont normalisées (Figure 7) par rapport au manteau primitif (McDonough et Sun, 1995). Les spectres des terres rares (Figure 7a) sont fortement pentus avec des valeurs des teneurs en terres rares légères élevées et comprises entre 50 et 170 fois celles du manteau primitif (Table 2). Ils sont caractérisés par un enrichissement en terres rares légères par rapport aux terres rares lourdes (10 < $\mathrm{LaN} / \mathrm{YbN}<23 ; 2,0<\mathrm{LaN} / \mathrm{SmN}<5,0)$. Les valeurs des rapports $\mathrm{CeN} / \mathrm{YbN}$ des laves sont élevées et comprises entre 10,0 et 18,0. De fortes anomalies négatives en $\mathrm{K}, \mathrm{Pb}$ et $\mathrm{Ti}$ caractérisent les spectres multiélémentaires des laves (Figure 7b).

Les données isotopiques au $\mathrm{Sr}, \mathrm{Nd}$ et $\mathrm{Pb}$ de quelques laves basaltiques représentatives sont présentées dans le Tableau 2. La valeur du rapport Sri du basalte NG105 (recalculée à $9 \mathrm{Ma}$ ) (Nkouandou et al., 2008) est de 0,7031. La mugéarite NG24 a une valeur Sri de 0,7033. Ces valeurs des rapports $\mathrm{Sr}$ sont typiques des roches d'origine mantellique. Les rapports isotopiques $\mathrm{Nd}^{143} / \mathrm{Nd}^{144}$ recalculés à $9 \mathrm{Ma}$ du basalte 
NG105 et de la mugéarite NG24 sont respectivement de 0,51289 et 0,51285 , pour les valeurs respectives de $\xi \mathrm{Nd}$ de $+5,03$ et $+4,39$. Les valeurs des rapports isotopiques du $\mathrm{Pb}$, notamment les rapports ${ }^{206} \mathrm{~Pb} /{ }^{204} \mathrm{~Pb}$, ${ }^{207} \mathrm{~Pb} /{ }^{204} \mathrm{~Pb}$ et ${ }^{208} \mathrm{~Pb} /{ }^{204} \mathrm{~Pb}$, sont respectivement de $19,976,15,686$ et 39,563 pour le basalte NG105 et respectivement de 19,985, 15,706 et 39,777 pour la mugéarite NG24. Toutes les valeurs des rapports isotopiques des laves basaltiques représentatives de Ngaoundéré appartiennent au domaine déterminé par les autres basaltes des secteurs océanique et continental de la 'Ligne Chaude du Cameroun' (Halliday et al., 1990; Déruelle et al., 2007) avec cependant des valeurs du néodyme relativement faibles. Celles du plomb sont élevées pour ${ }^{207} \mathrm{~Pb} /{ }^{204} \mathrm{~Pb}(\approx 15,7)$ par rapport à l'ensemble de la 'Ligne Chaude du Cameroun'.

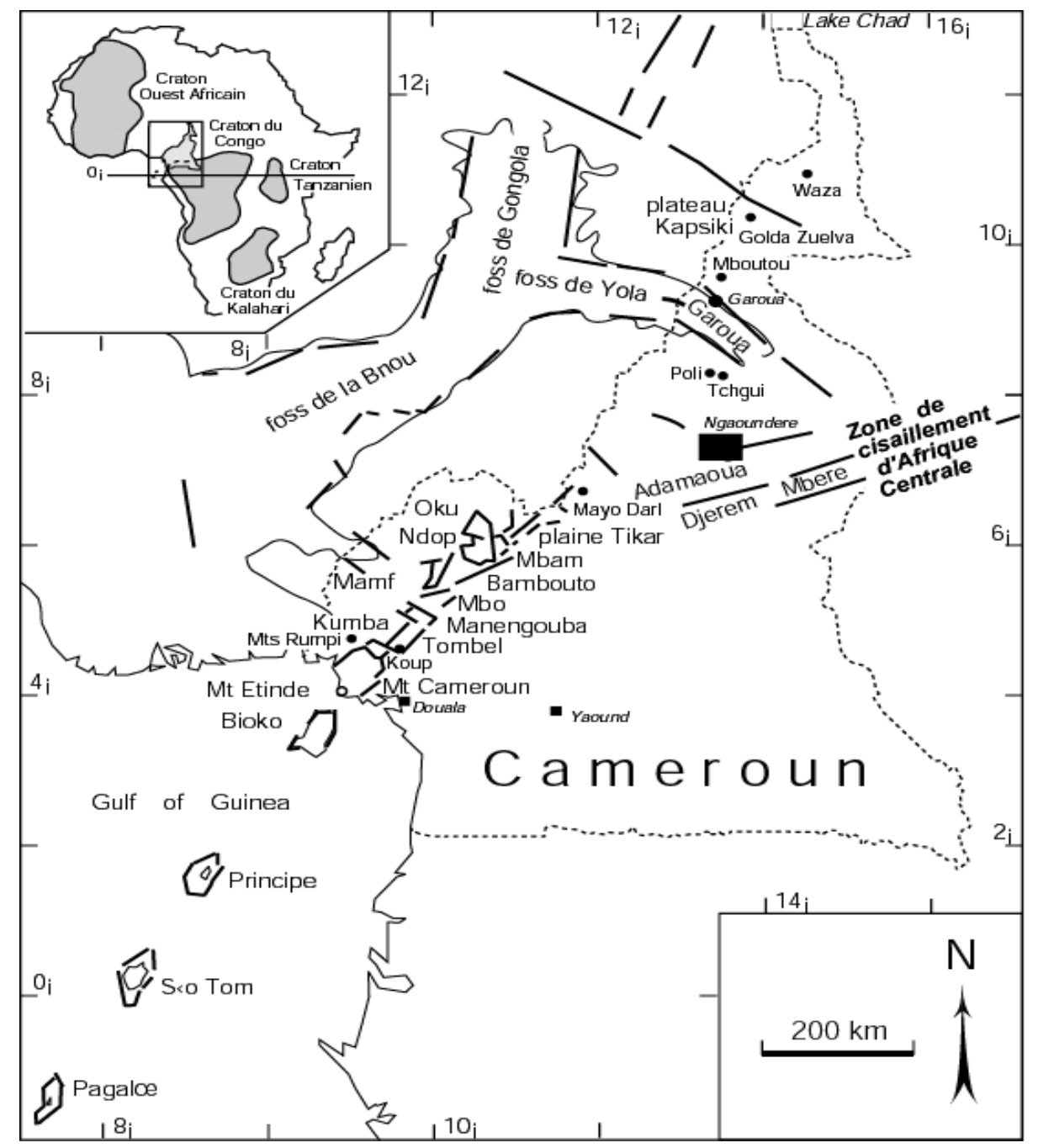

Figure 1: Le Plateau de l'Adamaoua, la 'Ligne du Cameroun' et le cisaillement d'Afrique centrale. La région de Ngaoundéré est représentée par le rectangle noir. 


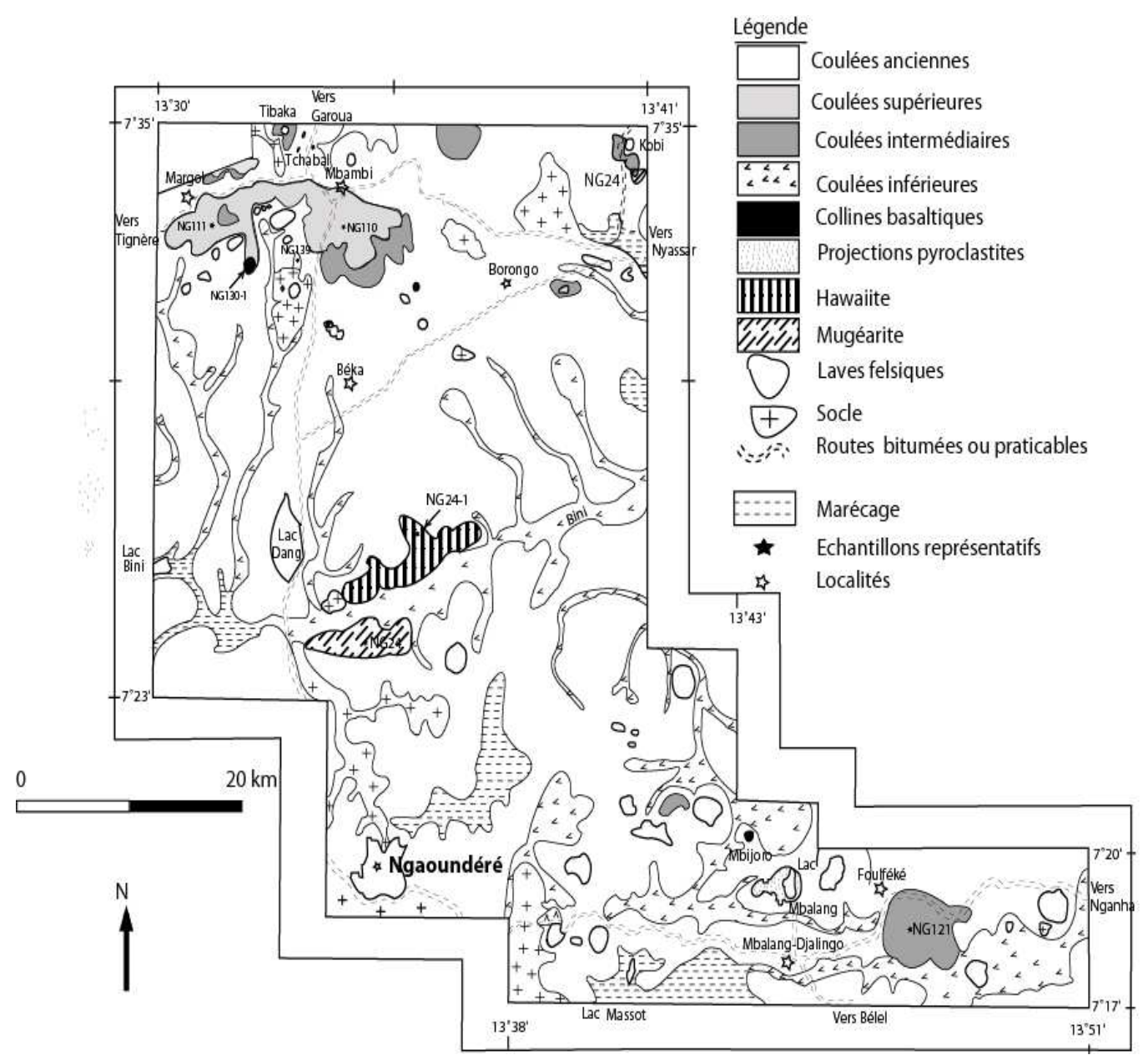

Figure 2 : Carte géologique des zones Nord et Est de Ngaoundéré.

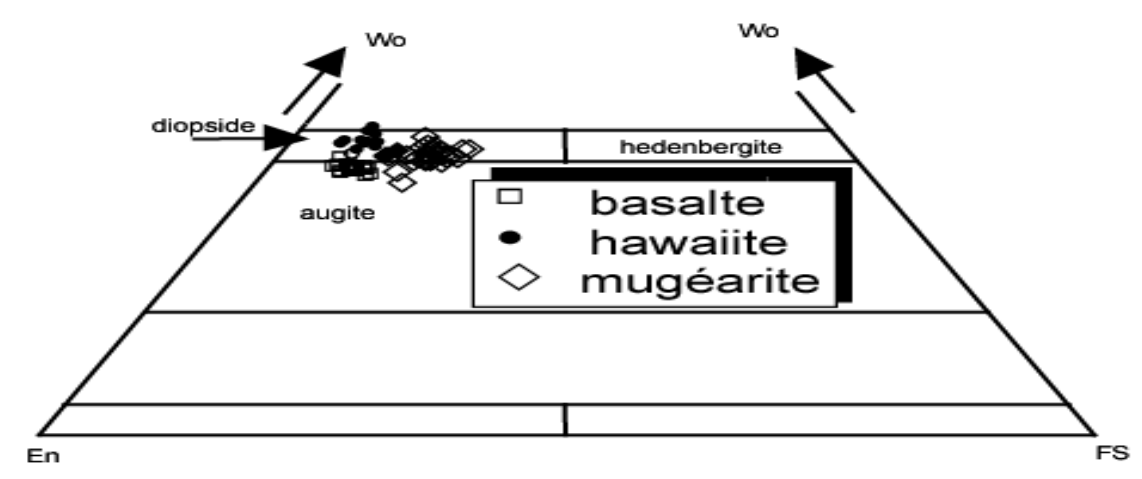

Figure 3: Compositions des cristaux de clinopyroxène des laves dans le diagramme Di-En-Fs d'après Morimoto et al. (1988). 


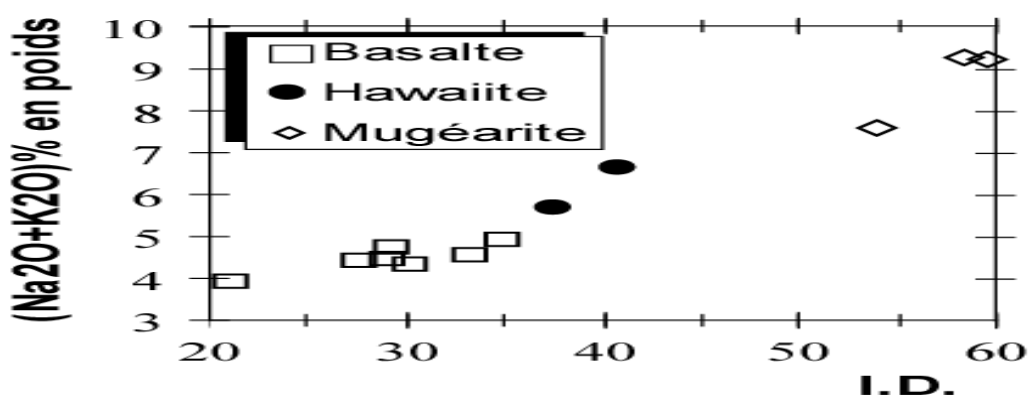

Figure 4: Nomenclature des laves des zones Nord et Est de Ngaoundéré. I.D.: indice de différenciation (d'après Thornton et Tuttle, 1960).

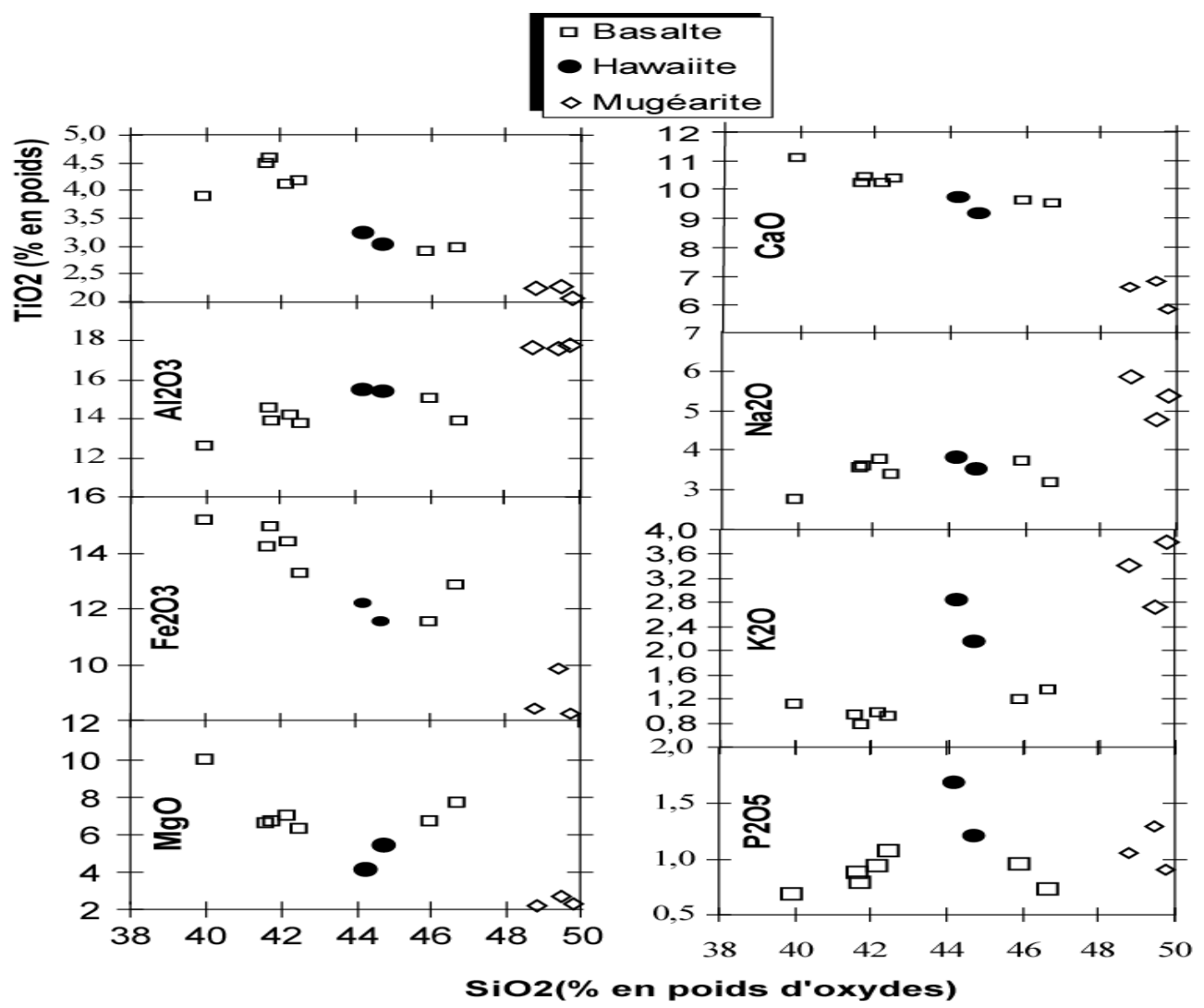

Figure 5: Diagramme de distribution des éléments majeurs en fonction des teneurs de $\mathrm{SiO}_{2}$ (en poids \%). 


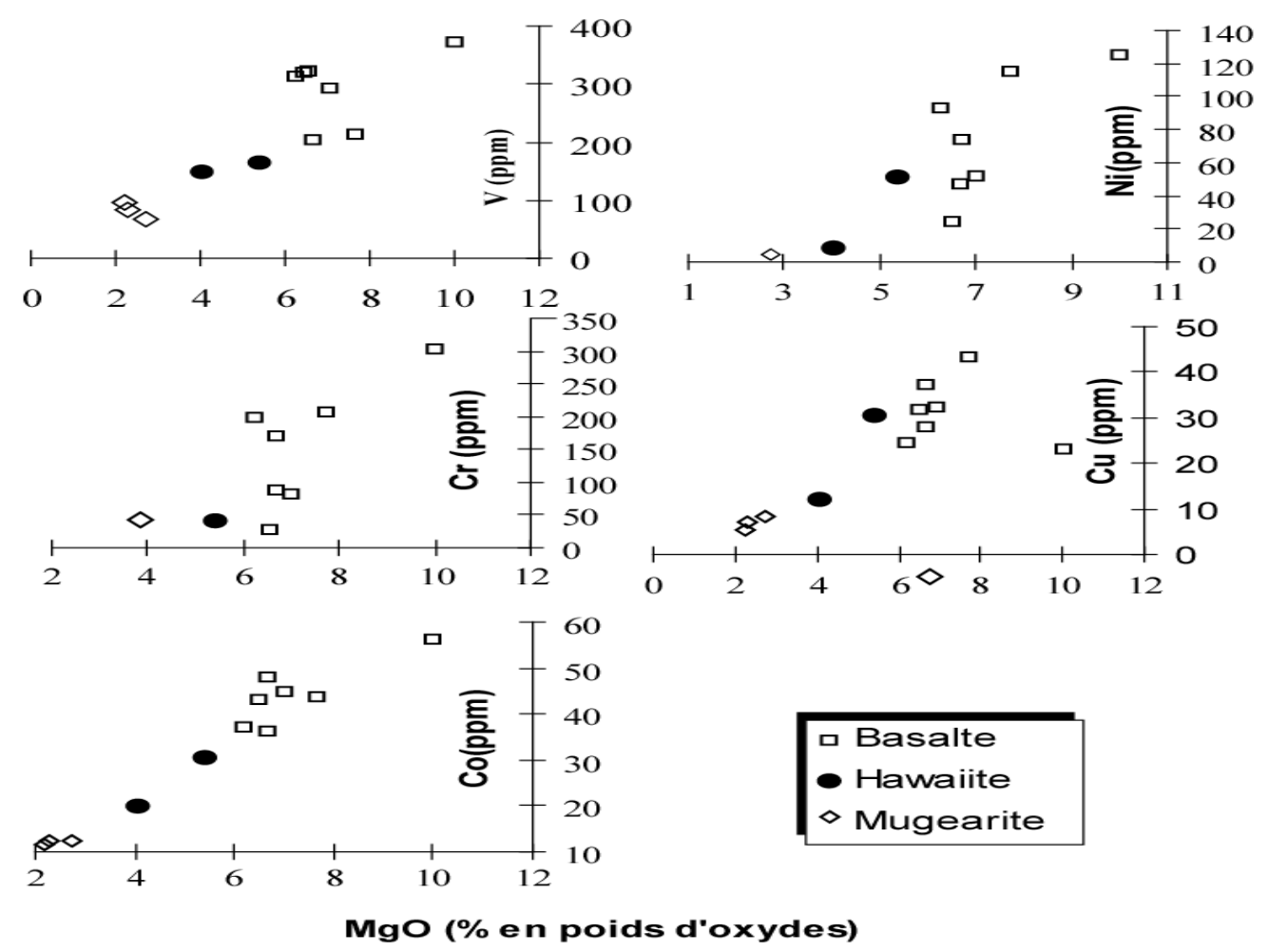

Figure 6: Diagramme de distribution des éléments de transition (ppm) en fonction des teneurs en poids $\%$ de $\mathrm{MgO}$.

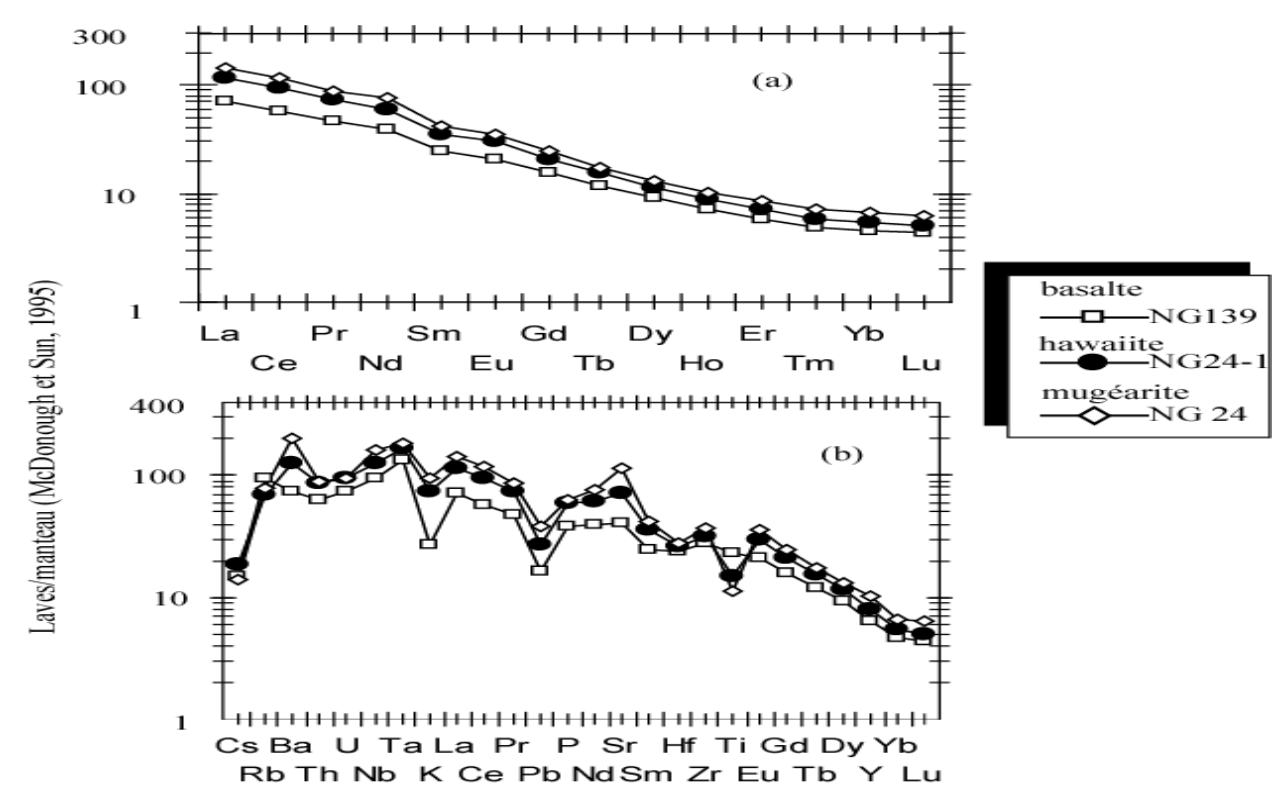

Figure 7: Spectres des terres rares (a) et des éléments en trace (b) des laves normalisées au manteau primitif (McDonough et Sun, 1995). 


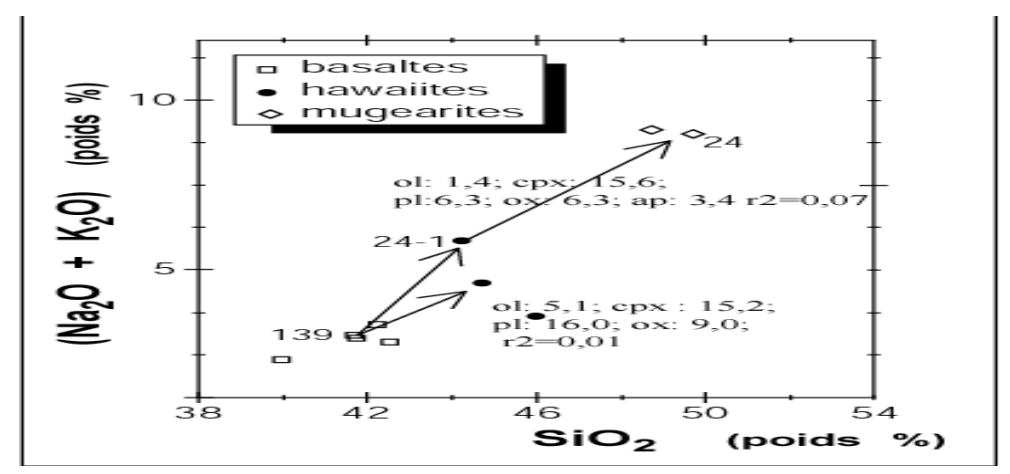

Figure 8: Test de différenciation des laves basaltiques de Ngaoundéré par fractionnement des éléments majeurs en système fermé.

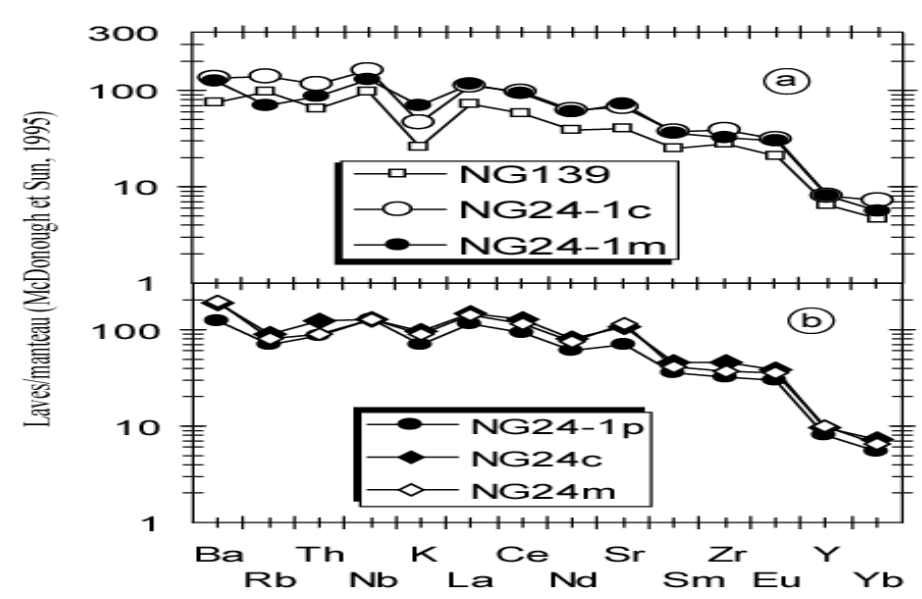

Figure 9: Modèle de différenciation des laves par fractionnement des éléments en système clos. (a): différenciation basalte $\longrightarrow$ hawaiite et (b): hawaiite $\longrightarrow$ mugéarite.

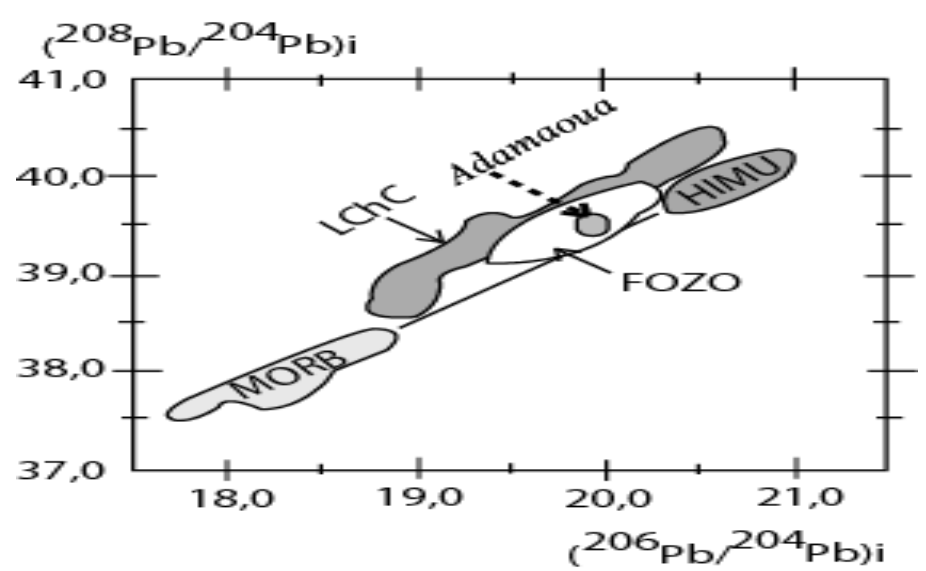

Figure 10: Diagramme (208Pb/206Pb)i-(208Pb/204Pb)i des laves basaltiques de Ngaoundéré dans le champ FOZO (rond gris). 
O.F. NKOUANDOU et al. / Int. J. Biol. Chem. Sci. 4(4): 984-1003, 2010

Tableau 1: Analyses chimiques des minéraux représentatifs des laves.

\begin{tabular}{|c|c|c|c|c|c|c|c|c|c|c|}
\hline Minéraux & ol & & & & cpx & & & & $\mathrm{mt}$ & ilm \\
\hline Laves & $\mathrm{ba}$ & & ha & mug & ba & ha & mug & $\mathrm{ba}$ & & \\
\hline Echantillons & NG139 & NG121 & NG24-1 & NG24 & NG130 & NG142 & NG14 & NG131 & & NG130 \\
\hline Poids (\%) & $\mathrm{Ph}, \mathrm{c}$ & & mic & $\mathrm{Ph}, \mathrm{c}$ & & & & & mic & \\
\hline $\mathrm{SiO}_{2}$ & 38,38 & 37,68 & 36,31 & 37,94 & 46,31 & 47,21 & 47,10 & 45,30 & 0,02 & 0,15 \\
\hline $\mathrm{TiO}_{2}$ & & & & & 2,44 & 1,95 & 2,55 & 3,17 & 24,32 & 39,46 \\
\hline $\mathrm{Al}_{2} \mathrm{O}_{3}$ & & & & & 6,49 & 6,81 & 6,78 & 7,81 & 1,79 & 0,15 \\
\hline $\mathrm{MnO}$ & 0,22 & 0,62 & 0,78 & 1,25 & 0,18 & 0,09 & 0,35 & 0,46 & 0,90 & 0,69 \\
\hline $\mathrm{MgO}$ & 41,02 & 33,35 & 33,32 & 32,38 & 13,04 & 13,37 & 11,00 & 9,50 & 3,11 & 5,37 \\
\hline $\mathrm{CaO}$ & 0,25 & 0,37 & 0,40 & 0,49 & 21,93 & 21,83 & 21,42 & 20,99 & 0,02 & 1,71 \\
\hline $\mathrm{NiO}$ & 0,04 & & 0,07 & & 0,00 & & & 0 & 0,05 & 0,10 \\
\hline $\mathrm{Na}_{2} \mathrm{O}$ & & & & & 0,58 & 0,96 & 1,10 & 1,40 & & \\
\hline Total & 99,31 & 99,89 & 99,89 & 99,72 & 99,11 & 99,60 & 99,32 & 99,32 & 98,02 & 97,63 \\
\hline $\mathrm{Ti}$ & & & & & 0,069 & 0,055 & 0,072 & 0,090 & 5,281 & 1,821 \\
\hline $\mathrm{Al}$ & & & & & 0,287 & 0,300 & 0,301 & 0,350 & 0,607 & 0,009 \\
\hline $\mathrm{Cr}$ & & & & & 0,003 & 0,005 & & 0,000 & 0,002 & \\
\hline $\mathrm{Fe} 3+$ & & & & & 0,136 & 0,128 & 0,091 & 0,140 & 4,638 & 0,334 \\
\hline $\mathrm{Fe} 2+$ & 0,417 & 0,626 & 0,656 & 0,630 & 0,105 & 0,098 & 0,192 & 0,198 & 11,702 & 1,311 \\
\hline $\mathrm{Mn}$ & 0,005 & 0,014 & 0,019 & 0,030 & 0,006 & 0,003 & 0,011 & 0,015 & 0,220 & 0,028 \\
\hline $\mathrm{Mg}$ & 1,579 & 1,335 & 1,344 & 1,270 & 0,730 & 0,744 & 0,617 & 0,537 & 1,337 & 0,393 \\
\hline $\mathrm{Ca}$ & 0,007 & 0,011 & 0,011 & 0,010 & 0,882 & 0,834 & 0,863 & 0,852 & 0,006 & 0,090 \\
\hline $\mathrm{Ni}$ & & & & & & & & & 0,002 & 0,004 \\
\hline
\end{tabular}


O.F. NKOUANDOU et al. / Int. J. Biol. Chem. Sci. 4(4): 984-1003, 2010

\begin{tabular}{|c|c|c|c|c|c|c|c|c|c|}
\hline $\begin{array}{l}\mathrm{Na} \\
\mathrm{Fo}\end{array}$ & 78.9 & 68,1 & 66.6 & 65.9 & 0,043 & 0,070 & 0,080 & 0,103 & \\
\hline Wo & & & & & 46,0 & 44,0 & 46,4 & 47,0 & \\
\hline En & & & & & 49,0 & 50,0 & 41,1 & 39,0 & \\
\hline Fs & & & & & 5,0 & 6,0 & 12,6 & 14 & \\
\hline$(\%)$ & & & & & & & & & 67,2 \\
\hline Usp & & & & & & & & & \\
\hline
\end{tabular}

Tableau 1 (suite)

\begin{tabular}{|c|c|c|c|c|c|c|c|c|c|}
\hline Minéraux & $\mathrm{pl}$ & & & & $\begin{array}{l}\text { k-felds } \\
\text { (xéno) }\end{array}$ & & kaer & & ap \\
\hline Laves & ba & & ha & mug & ba & mug & ba & mug & \\
\hline Echantillons & NG139 & NG121 & NG24-1 & NG24 & NG130 & NG8 & NG130 & NG14 & \\
\hline Poids (\%) & $\mathrm{ml}$ & & & & $\mathrm{Ph}, \mathrm{c}$ & & & & \\
\hline $\mathrm{SiO}_{2}$ & 50,89 & 52,99 & 53,38 & 58,74 & 63,66 & 63,94 & 39,03 & 39,32 & 0,26 \\
\hline $\mathrm{TiO}_{2}$ & & & & & & & 5,99 & 5,53 & \\
\hline $\mathrm{Al}_{2} \mathrm{O}_{3}$ & 30,60 & 29,37 & 29,03 & 26,15 & 21,34 & 21,67 & 13,70 & 13,01 & \\
\hline $\mathrm{FeO}^{*}$ & 0,44 & 0,58 & 0,70 & 0,39 & 0,25 & 0,46 & 13,74 & 13,38 & 0,50 \\
\hline $\mathrm{MnO}$ & & & & & & & 0,26 & & \\
\hline $\mathrm{MgO}$ & & & & & & & 10,82 & 10,76 & \\
\hline $\mathrm{CaO}$ & 13,13 & 10,57 & 11,08 & 7,57 & 2,30 & 1,98 & 11,07 & 10,87 & 52,53 \\
\hline $\mathrm{Na}_{2} \mathrm{O}$ & 3,94 & 5,44 & 5,00 & 5,30 & 7,93 & 6,47 & 2,70 & 2,68 & 0,31 \\
\hline $\mathrm{K}_{2} \mathrm{O}$ & 0,22 & 0,31 & 0,30 & 0,91 & 3,16 & 5,31 & 1,01 & 1,43 & \\
\hline $\mathrm{P}_{2} \mathrm{O}_{5}$ & & & & & & & & & 40,79 \\
\hline $\mathrm{BaO}$ & & 0,15 & & 0,08 & 0,57 & & & & 0,21 \\
\hline $\mathrm{F}$ & & & & & & & 0,52 & 0,07 & 2,67 \\
\hline
\end{tabular}


O.F. NKOUANDOU et al. / Int. J. Biol. Chem. Sci. 4(4): 984-1003, 2010

\begin{tabular}{lccccccccc}
$\mathrm{Cl}$ & & & & & & & & & 0,02 \\
$\mathrm{Total}$ & 99,22 & 99,42 & 99,60 & 100,06 & 99,21 & 99,78 & 99,06 & 97,37 & 97,06 \\
$\mathrm{Fe}_{2} \mathrm{O}_{3}$ & 0,49 & 0,64 & & & & & 12,53 & 0,22 & \\
$\mathrm{FeO}$ & & & & & & & 1,85 & 13,68 & \\
$\mathrm{Total}$ (calc) & 99,27 & 99,49 & 99,60 & 100,06 & 99,21 & 99,78 & 99,70 & 97,89 & 97,06 \\
$\mathrm{Si}$ & 2,309 & 2,435 & 2,400 & 2,627 & 2,863 & 2,872 & 5,791 & 5,923 & 0,045 \\
$\mathrm{Ti}$ & & & & & & & 0,669 & 0,627 & \\
$\mathrm{Al}$ & 1,669 & 1,536 & 1,570 & 1,378 & 1,131 & 1,094 & 2,400 & 2,310 & \\
$\mathrm{Fe} 3+$ & 0,017 & 0,022 & 0,030 & & & & 0,200 & & \\
$\mathrm{Fe}+$ & & & & & & & 1,505 & 1,662 & 0,072 \\
$\mathrm{Mn}$ & & & & & & & 0,033 & 0,038 & \\
$\mathrm{Mg}$ & 0,651 & 0,521 & 0,550 & 0,363 & 0,111 & 0,093 & 1,759 & 1,754 & 5,979 \\
$\mathrm{Ca}$ & 0,354 & 0,486 & 0,450 & 0,548 & 0,693 & 0,565 & 0,778 & 0,783 & \\
$\mathrm{Na}$ & 0,013 & 0,018 & 0,020 & 0,052 & 0,181 & 0,304 & 0,192 & 0,275 & 0,002 \\
$\mathrm{~K}$ & & & & & & & & & 5,979 \\
$\mathrm{P}$ & & 0,003 & & & 0,010 & & & & 0,013 \\
$\mathrm{Ba}$ & & & & & & & 1,478 & & \\
$\mathrm{~F}$ & 1,3 & 1,8 & 2,0 & 5,4 & 18,4 & 31,6 & & & \\
$\mathrm{Or}$ & 34,8 & 47,4 & 44,0 & 56,9 & 70,4 & 58,7 & & & \\
$\mathrm{Ab}$ & 63,9 & 50,8 & 54,0 & 37,7 & 11,2 & 9,7 & & & \\
$\mathrm{An}$ & & & & & & & 61,4 & 59,2 & \\
$\mathrm{Mg \#}$ & & & & & & & & \\
\hline olivine, cpx: clinopyroxène, k-felds: feldspath potassique, pl: plagioclase, ox: oxydes, kaers: kaersutite et ap: apatite.
\end{tabular}

ol: olivine, cpx: clinopyroxène, k-felds: feldspath potassique, pl: plagioclase, ox: oxydes, kaers: kaersutite et ap: apatite.
ph,c: phénocristaux cœur; mic: microcristaux; ml: microlite. Xéno: xénocristaux; ba: basalte; ha: hawaiite; mug: mugéarite 
Tableau 2 : Analyses chimiques (majeurs, traces et isotopes) représentatives des laves basaltiques de Ngaoundéré.

\begin{tabular}{|c|c|c|c|}
\hline Laves & basalte & hawaiite & mugéarite \\
\hline Echantillons & NG105 & NG24-1 & NG24 \\
\hline $\mathrm{SiO}_{2}$ (poids \%) & 41,66 & 44,70 & 49,47 \\
\hline $\mathrm{TiO}_{2}$ & 54,50 & 3,01 & 2,28 \\
\hline $\mathrm{Al}_{2} \mathrm{O}_{3}$ & 14,59 & 15,39 & 17,59 \\
\hline $\mathrm{Fe}_{2} \mathrm{O}_{5}$ & 14,23 & 12,20 & 9,85 \\
\hline $\mathrm{MnO}$ & 0,20 & 0,21 & 0,26 \\
\hline $\mathrm{MgO}$ & 6,57 & 5,42 & 2,74 \\
\hline $\mathrm{CaO}$ & 10,20 & 9,17 & 6,82 \\
\hline $\mathrm{Na}_{2} \mathrm{O}$ & 3,55 & 3,53 & 4,83 \\
\hline $\mathrm{K}_{2} \mathrm{O}$ & 0,92 & 2,14 & 2,74 \\
\hline $\mathrm{P}_{2} \mathrm{O}_{5}$ & 0,87 & 1,21 & 1,29 \\
\hline LOI & 1,78 & 2,44 & 1,05 \\
\hline Total & 99,07 & 99,45 & 98,92 \\
\hline $\mathrm{Ne}$ & 7,39 & 6,00 & 3,72 \\
\hline D.I. & 29,18 & 37,44 & 53,92 \\
\hline $\mathrm{Be}(\mathrm{ppm})$ & 1,9 & 1,9 & 2,3 \\
\hline $\mathrm{Rb}$ & 45 & 42 & 48 \\
\hline $\mathrm{Sr}$ & 967 & 1413 & 2256 \\
\hline Cs & 0,44 & 0,39 & 0,30 \\
\hline $\mathrm{Ba}$ & 564 & 830 & 1312 \\
\hline V & 319 & 163 & 67 \\
\hline $\mathrm{Cr}$ & 25,5 & 41,0 & 5,3 \\
\hline Co & 43,1 & 30,3 & 12,6 \\
\hline $\mathrm{Ni}$ & 23,6 & 50,9 & 4,8 \\
\hline $\mathrm{Cu}$ & 31 & 30 & 8 \\
\hline $\mathrm{Zn}$ & 159 & 154 & 170 \\
\hline $\mathrm{Ga}$ & 26 & 24 & 24 \\
\hline $\mathrm{Y}$ & 32,1 & 34,3 & 43,7 \\
\hline $\mathrm{Zr}$ & 465 & 333 & 387 \\
\hline $\mathrm{Nb}$ & 87 & 84 & 106 \\
\hline Hf & 9,3 & 7,4 & 7,8 \\
\hline $\mathrm{Ta}$ & 5,86 & 6,14 & 6,79 \\
\hline Th & 6,12 & 6,93 & 7,1 \\
\hline $\mathrm{U}$ & 1,77 & 1,93 & 1,91 \\
\hline $\mathrm{Pb}$ & 3,18 & 4,09 & 5,72 \\
\hline $\mathrm{La}$ & 53,1 & 75,4 & 93,6 \\
\hline $\mathrm{Ce}$ & 115 & 157 & 196 \\
\hline $\operatorname{Pr}$ & 12,8 & 18,9 & 22,1 \\
\hline $\mathrm{Nd}$ & 56 & 75 & 94 \\
\hline $\mathrm{Sm}$ & 11,4 & 14,5 & 17,0 \\
\hline $\mathrm{Eu}$ & 3,56 & 4,63 & 5,50 \\
\hline
\end{tabular}




\begin{tabular}{lccc}
$\mathrm{Gd}$ & 9,67 & 11,40 & 13,27 \\
$\mathrm{~Tb}$ & 1,314 & 1,536 & 1,733 \\
$\mathrm{Dy}$ & 6,88 & 7,78 & 8,84 \\
$\mathrm{Ho}$ & 1,16 & 1,30 & 1,50 \\
$\mathrm{Er}$ & 2,91 & 3,19 & 3,74 \\
$\mathrm{Tm}$ & 0,37 & 0,40 & 0,48 \\
$\mathrm{Yb}$ & 2,29 & 2,43 & 2,92 \\
$\mathrm{Lu}$ & 0,34 & 0,34 & 0,43 \\
$\left.{ }^{87} \mathrm{Sr} /{ }^{86} \mathrm{Sr}\right)_{9 \mathrm{Ma}}$ & 0,7031 & & 0,7033 \\
$\xi_{\mathrm{Nd} 9 \mathrm{Ma}}$ & 5,03 & & 4,39 \\
${ }^{206} \mathrm{~Pb} /{ }^{204} \mathrm{~Pb}$ & 19,976 & & 19,985 \\
${ }^{207} \mathrm{~Pb} /{ }^{204} \mathrm{~Pb}$ & 15,686 & & 15,706 \\
${ }^{208} \mathrm{~Pb} /{ }^{204} \mathrm{~Pb}$ & 39,563 & & 39,777 \\
\hline
\end{tabular}

\section{DISCUSSION}

Pétrogenèse des laves basaltiques récentes des zones Nord et Est de Ngaoundéré - Cristallisation fractionnée

Des modèles de fractionnement magmatique en système fermé (Shaw, 1970; DePaolo, 1981) ont été proposés pour expliquer la différenciation et l'évolution des séries magmatiques. Pour les laves basaltiques de Ngaoundéré, les compositions des éléments majeurs et traces seront utilisées pour tester l'évolution en système fermé des laves par cristallisation fractionnée.

\section{Éléments majeurs}

Les corrélations observées (Figures 5 et 6) pour les laves des régions au Nord et à l'Est de Ngaoundéré sont typiques des séries magmatiques alcalines. Les corrélations linéaires et la forte décroissance des teneurs en éléments de transition au cours de la différenciation (Figures 5 et 6 ) sont des arguments en faveur d'une différenciation par cristallisation fractionnée des laves basaltiques de Ngaoundéré.

Des mélanges magmatiques ont aussi pu intervenir dans la genèse des basaltes et des mugéarites comme en témoigne la présence des cristaux de sanidine dans les basaltes de Voglar et des cristaux d'olivine (Fo66) et surtout de titanite dans les mugéarites. Un test (Figure 8) de la différenciation magmatique par cristallisation des phases minérales présentes en phénocristaux dans les laves a été effectué (données en \% en masse). Les calculs sont effectués sur la base d'un bilan de masse en minimisant la somme des carrés de résidus : basalte (ol: 5,1; cpx: 15,2; pl: 16,0; ox: 9,0; $\left.\sum \mathrm{r} 2=0,01\right) \longrightarrow$ hawaiite (ol: 1,4; cpx: 15,6; pl: 6,3; ox: 6,3; ap: $\left.3,4 \sum \mathrm{r} 2=0,07\right) \longrightarrow$ mugéarite. Les sommes des carrés des résidus $\left(\sum \mathrm{r} 2\right)$ sont faibles.

\section{Éléments traces}

Le test de la cristallisation fractionnée par fractionnement des éléments traces en système fermé est proposé en utilisant l'équation classique de Rayleigh: $\mathrm{C}_{1}^{\mathrm{i}}=\mathrm{C}_{\mathrm{o}}^{\mathrm{i}} \mathrm{F}^{(\mathrm{Di} \text { - }}$ 1) ( $\mathrm{Cl}^{\mathrm{i}}$ est la concentration de l'élément "i" dans le liquide calculé "l"; $\mathrm{Co}^{\mathrm{i}}$ est la concentration de "i" dans le magma parent; $\mathrm{F}$ est la proportion du liquide parental fractionné et $\mathrm{Di}$, le coefficient de distribution de l'élément "i"). Les coefficients de partage utilisés dans le calcul de Di sont tirés de Green et al. (1994). Les proportions des phases minérales utilisées sont celles obtenues dans les calculs de la modélisation par les éléments majeurs. Les spectres multiélémentaires normalisés au manteau primitif (McDonough et Sun, 1995) des laves calculées et mesurées sont présentés (Figure 
9). D'après ce modèle, l'hawaiite NG24-1 dérive du basalte NG139. En dehors du Sr qui a un rapport $\mathrm{Srm} / \mathrm{Src}$ de 0,87 , tous les éléments traces donnent des rapports NG24$1 \mathrm{~m} / \mathrm{NG} 24-1 \mathrm{c}$ très proches de l'unité. Seul K est déficitaire. La mugéarite NG24 dérive de l'hawaiite NG24-1. Pour cette phase de différenciation, les rapports $\mathrm{m} / \mathrm{c}$ de $\mathrm{Sr}$ et $\mathrm{Ba}$ sont légèrement excédentaires (1,26 et 1,29 respectivement). Les résultats de ce test sont en accord avec une différenciation des magmas par cristallisation fractionnée. Les rapports $\mathrm{m} / \mathrm{c}$ excédentaires ou déficitaires de certains éléments, peuvent s'expliquer par les variations des valeurs de $\mathrm{Kd}$ ou par l'implication des processus autres que ceux de la cristallisation fractionnée au cours de l'évolution des laves.

\section{Nature de la source et origine des basaltes Caractéristiques chimiques}

Il est communément admis que les basaltes alcalins peu évolués ont des concentrations en éléments de transition de la première série relativement constantes $\left(\mathrm{C}^{\mathrm{Co}}=\right.$ $60 \pm 10$ ppm; $C^{\mathrm{Ni}}=350 \pm 150$ ppm) (Allègre et Minster, 1978; Villemant et Treuil, 1983). De telles concentrations sont attribuées à une origine par fusion partielle d'une source mantellique péridotitique.

Les faibles teneurs en $\mathrm{Ni}$ (124 ppm) et $\mathrm{MgO}(7 \%)$ des basaltes révèlent le caractère évolué de leur magma parent. Il n'est donc pas possible d'assimiler cette composition à celle d'un magma parent issu de la fusion partielle d'une source mantellique lherzolitique (riche en $\mathrm{Fo}_{90}$ ) avec 400 ppm de $\mathrm{Ni}$ (Green et al., 1974), à moins que celui-ci n'ait lui-même subi une cristallisation précoce d'olivine l'ayant appauvri en $\mathrm{Mg}$ et $\mathrm{Ni}$. Les valeurs élevées des rapports $\mathrm{CeN} / \mathrm{YbN}$ des basaltes (entre 13,0 et 18,0) sont de l'ordre de celles qui caractérisent les basaltes du domaine continental de la "Ligne Chaude du Cameroun" $\left(12<\mathrm{Ce}_{\mathrm{N}} / \mathrm{Yb}_{\mathrm{N}}<22\right.$, (Ngounouno et al., 2003 et 2006). Elles suggèrent des faibles taux de fusion du manteau source. Un faible taux de fusion (1 à 2\%) d'un manteau proche du type HIMU composé de $79 \%$ MORB+18\% DM+1\% sédiment pélagique a été proposé (Nkouandou, 2006) pour la genèse des basaltes de Ngaoundéré.

Les spectres normalisés des basaltes de la région de Ngaoundéré (Figure 7) sont caractérisés par des anomalies négatives en $\mathrm{K}$ et $\mathrm{Pb}$. Il a été suggéré que l'anomalie négative en $\mathrm{K}$ est une caractéristique de la source des basaltes et qu'elle pourrait résulter de l'existence de phlogopite résiduelle lors d'une fusion partielle à faible taux du manteau source (Dupuy et al., 1988 ; Weaver, 1990 ; Chauvel et al., 1992). Les coefficients de distribution de $\mathrm{K}$ sont nettement supérieurs à 1 dans les systèmes basaltiques à phlogopite (Green et al., 1994). Le déficit en $\mathrm{Pb}$ a été interprété comme résultant de l'altération hydrothermale de la croûte océanique lors de sa genèse et/ou d'une déshydratation lors de sa subduction (Chauvel et al., 1992), tout comme les anomalies positives en $\mathrm{Nb}$ et $\mathrm{Ta}$ des spectres multiélémentaires qui pourraient résulter d'un enrichissement de la source mantellique en ces éléments (Weaver, 1991). Les basaltes de Ngaoundéré proviendraient donc, comme ceux des îles océaniques de type HIMU (et vraisemblablement pour une source magmatique de type FOZO), d'un faible taux de fusion partielle (1 à 2\%) avec phlogopite résiduelle d'un manteau enrichi en $\mathrm{Pb}, \mathrm{Nb}$ et Ta lors d'une subduction. Une subduction vers l'Est le long du craton congolais (Est du Tchad-Nord du Cameroun) caractérise l'orogenèse entre 800 et 630 M.a. (Toteu et al., 1990) qui, fertilisant le manteau, peut être à l'origine du caractère HIMU des sources magmatiques des laves basaltiques de Ngaoundéré. Les données isotopiques des laves basaltiques de Ngaoundéré qui caractérisent une source mantellique de composition proche du champ HIMU (Zindler et Hart, 1986) sont des arguments en faveur de cette hypothèse. 


\section{Caractéristiques isotopiques: une source mantellique de type $\mathrm{FOZO}$}

Les basaltes appartenant au domaine FOZO ont des caractéristiques isotopiques intermédiaires entre celles des basaltes représentant les quatre pôles mantelliques précédemment définis par Hart et al. (1992). Les sources magmatiques mantelliques de type FOZO (FOcal ZOne) telles que définies par Hart et al. (1992) sont caractérisées par une dynamique de composition et de grande variation des valeurs des rapports isotopiques de $\mathrm{Sr}$, Nd et $\mathrm{Pb}$ (Stracke et al., 2005). Pour les laves basaltiques de Ngaoundéré, les valeurs des rapports isotopiques du $\mathrm{Sr}$ recalculées à 9 Ma (âge moyen des laves basaltiques), 0,7031 $\left.<{ }^{87} \mathrm{Sr} /{ }^{86} \mathrm{Sr}\right) \mathrm{i}>0,7034$ (Nkouandou et al., 2008) sont semblables à celles des autres régions volcaniques du plateau de l'Adamaoua (Nono et al., 1994) et des secteurs océanique et continental de la 'Ligne Chaude du Cameroun' (Halliday et al., 1988 ; Ngounouno et al., 2003 ; Déruelle et al., 2007). Les valeurs des rapports isotopiques $\mathrm{Pb}-\mathrm{Pb}$, en particulier ${ }^{208} \mathrm{~Pb} /{ }^{204} \mathrm{~Pb}$ des laves basaltiques de Ngaoundéré $\left(39,56<{ }^{208} \mathrm{~Pb} /{ }^{204} \mathrm{~Pb}<39,40\right) \quad$ sont également comprises dans l'intervalle de celles de la 'Ligne Chaude du Cameroun' à Pagalu $\left({ }^{208} \mathrm{~Pb} /{ }^{204} \mathrm{~Pb}=38,83\right)$, à Bioko, au Mt Etindé et au Mt Cameroun $\left({ }^{208} \mathrm{~Pb} /{ }^{204} \mathrm{~Pb}<40,34\right)$ étudiées par Déruelle et al. (2007). Elles sont similaires à celles des sources magmatiques mantelliques de type Focal Zone (FOZO): ${ }^{206} \mathrm{~Pb} /{ }^{204} \mathrm{~Pb}=19,5-20,5,{ }^{87} \mathrm{Sr} /{ }^{86} \mathrm{Sr}=0,7028$ 0,7034 et ${ }^{143} \mathrm{Nd} /{ }^{144} \mathrm{Nd}=0,5128-0,5130$, récemment définies par Stracke et al. (2005).

Dans le diagramme ${ }^{208} \mathrm{~Pb} /{ }^{204} \mathrm{~Pb}$ vs ${ }^{206} \mathrm{~Pb} /{ }^{204} \mathrm{~Pb}$ (Figure 10), les valeurs des rapports isotopiques ${ }^{208} \mathrm{~Pb} /{ }^{204} \mathrm{~Pb}$ des laves basaltiques de Ngaoundéré, moins radiogéniques, sont très proches de celui de l'ensemble de la LChC et sont situées dans le champ FOZO par rapport au champ HIMU pour lequel elles sont plus radiogéniques. Il est donc possible d'envisager une source magmatique commune pour le volcanisme de
l'Adamaoua et de la LChC ou bien deux sources magmatiques de même composition pour ces deux régions.

\section{Origine des hawaiites et mugéarites Origine des hawaiites}

L'origine des hawaiites de la région de Ngaoundéré est discutée en termes de cristallisation fractionnée. Les spectres des hawaiites montrent des variations semblables à celles des basaltes mais ont des teneurs élevées en éléments en traces. Ils présentent des anomalies positives en $\mathrm{Ba}, \mathrm{Sr}$ et $\mathrm{Eu}$ et des anomalies négatives en $\mathrm{Cs}, \mathrm{K}, \mathrm{Pb}$ et $\mathrm{Ti}$. Les anomalies positives en $\mathrm{Ba}, \mathrm{Sr}$ et $\mathrm{Eu}$, éléments à coefficients de partage élevés pour le plagioclase (Green et al., 1994) traduisent certainement un très faible taux de cristallisation de ce minéral tel que suggère l'augmentation des teneurs en $\mathrm{Ba}$ et $\mathrm{Sr}$ des basaltes aux mugéarites (Tableau 2), en l'absence des phénocristaux de plagioclase. Les calculs effectués sur la base d'un bilan de masse en minimisant la somme des carrés de résidus suggèrent que les hawaiites proviennent du basalte NG139 par cristallisation fractionnée de 55,0\% d'olivine, $\mathrm{du}$ clinopyroxène $(15,2 \%)$, du plagioclase $(16,0 \%)$ et de la titanomagnétite $(9,0 \%)$. Le test de la cristallisation fractionnée par le fractionnement des éléments traces en système fermé soutient cette hypothèse.

\section{Origine des mugéarites}

Les bordures résorbées et parfois corrodées avec des nombreuses inclusions vitreuses dans certains cristaux de clinopyroxène et de feldspath des mugéarites et, la présence des cristaux d'olivine (Fo66) et de titanite dans cette lave sont des témoins de mélange magmatique. Des mélanges semblables ont été suggérés pour les mugéarites de la vallée de la Benoué (Ngounouno et al., 2000) et les benmoréites de Tchabal Djinga (Ezangono et al., 1995). Les spectres des mugéarites sont caractérisés par des anomalies négatives en $\mathrm{P}$ et très poussées en $\mathrm{Ti}$, suggérant la cristallisation 
fractionnée d'apatite, de kaersutite et d'oxydes de fer-titane. Les résultats des modélisations de la cristallisation fractionnée par les éléments majeurs et en traces (bilan de masse en système fermé) indiquent que les mugéarites pourraient être le produit de la cristallisation fractionnée des hawaiites (Figures 8 et 9). Le mélange de magma évoqué aurait une faible influence et la cristallisation fractionnée serait le processus majeur dans la genèse des mugéarites.

\section{Source métasomatisée}

La présence des phases riches en volatiles (kaersutite) décrites dans les basaltes, en conformité avec les travaux expérimentaux (Olafsson et Eggler, 1983), suggère que les magmas parentaux à l'origine des laves basaltiques de Ngaoundéré proviennent d'une source mantellique métasomatisée. Les faibles valeurs des teneurs en $\mathrm{Sr}(<30 \mathrm{ppm})$ et des rapports $\mathrm{Rb} / \mathrm{Sr} \quad(<0,05)$ des basaltes de Ngaoundéré caractérisent une source mantellique métasomatisée comme en atteste la présence des poches de carbonate dans les échantillons de basalte. Le phénomène métasomatique se serait produit postérieurement à la fusion partielle comme il a été mis en évidence par l'étude de quelques xénolithes du Cameroun (Lee et al., 1996). La source magmatique des laves basaltiques de Ngaoundéré serait donc située dans le manteau sous lithosphérique comme cela a été proposé pour les autres basaltes de la 'Ligne du Cameroun' (Moreau et al., 1987) c'est-àdire, juste en dessous de la limite croûtemanteau, entre 20 et $30 \mathrm{~km}$ (Poudjom Djomani et al., 1997).

\section{Contamination crustale}

Les teneurs en $\mathrm{P}_{2} \mathrm{O}_{5}$ des laves basaltiques de Ngaoundéré $\left(0,9<\mathrm{P}_{2} \mathrm{O}_{5}<1,3\right)$ sont semblables à celles des laves des secteurs continental (Kapsiki, 0,6 $<\mathrm{P}_{2} \mathrm{O}_{5}<1,2$ ) (Ngounouno et al., 2000) et océanique (0,4 < $\left.\mathrm{P}_{2} \mathrm{O}_{5}<1,4\right)$ (Fitton, 1987) de la 'Ligne Chaude du Cameroun', qui n'ont pas connu une contribution de matériel crustal. Une contamination de la source ne peut non plus être envisagée pour les éléments traces comme en attestent les anomalies positives de $\mathrm{Nb}$ et Ta des spectres multiélémentaires et les données isotopiques. Cependant, le basalte NG121 (Zr: 213 ppm, Nb: 43 ppm, Hf: 5 ppm, Ta: 3,21 ppm, Th: 3,45 ppm et U: 0,91 ppm) qui se distingue par des teneurs relativement faibles de certains éléments traces suggère des taux de fusion élevés par rapport aux autres laves basaltiques ou une contribution de la croûte continentale. Les données isotopiques ont donc été préservées et la contamination (pour certains éléments) dans ce cas est restée sélective. Le mélange magmatique suggéré (présence des xénocristaux dans les basaltes et les mugéarites) et la contamination sélective restent donc dominés par le processus de cristallisation fractionnée au cours de l'évolution des basaltes aux mugéarites.

\section{Conclusion}

Les données pétrographiques, minéralogiques et géochimiques (éléments majeurs, traces, terres rares et isotopiques) des laves basaltiques de Ngaoundéré sont caractéristiques d'un magmatisme franchement alcalin dont la différenciation est dominée par le processus de cristallisation fractionnée. Des mélanges magmatiques et une contamination sélective ont influencé la différenciation. Les données isotopiques déterminent une source magmatique mantellique de type Focal-Zone (FOZO), située dans le manteau sous lithosphérique comme cela a été proposé pour les autres basaltes de la 'Ligne du Cameroun', en dessous de la limite croûte-manteau, entre 20 et $30 \mathrm{~km}$. Une faible fusion partielle de cette source probablement métasomatisée aurait eu lieu lors d'une décompression adiabatique pendant la surrection du horst de l'Adamaoua.

\section{REMERCIEMENTS}

Les auteurs remercient le ministère français de Coopération à travers le Service de 
Coopération et d'Actions Culturelles de l'ambassade de France à Yaoundé au Cameroun, pour avoir permis à O.F.N. d'effectuer deux séjours en France à l'Université Pierre et Marie Curie, et pour l'aide financière à lui accordée pour les mesures isotopiques réalisées à l'Université Libre de Bruxelles. Les gratitudes des auteurs vont au Prof. A. Jambon pour avoir accordé des séances supplémentaires de sonde à $\mathrm{Mr}$. O.F.N (service CAMPARIS). Au Dr. Pierre Kamgang, qui a contribué à l'amélioration de ce manuscrit par ses critiques remarquables. Ces travaux sont une partie de la thèse de $\mathrm{Mr}$. O.F.N. soutenue à l'Université Pierre et Marie Curie (Paris VI).

\section{REFERENCES}

Allègre CJ, Minster JF. 1978. Quantitative models of trace elements behaviour in magmatic processes. Earth Planet. Sc. Lett., 38: 1-25.

Brown SE, Fairhead JD. 1983. Gravity study of the central African rift system: a model of continental disruption 1. The Ngaoundere and Abu Gabra rifts. Tectonophysics, 94: 187-203.

Carignan JP, Hild G, Mévelle J, Morel D. 2001. Yeghicheyan, Routine analyses of trace elements in geological samples using flow injection and low pressure on-line liquid chromatography coupled to ICP-MS: a study of geochemical reference materials $\mathrm{BR}$, DR-N, UB-N, AN-G and GH Geostandards. Newsletter, 25 : 187-198.

Chauvel C, Hofmann AW, Vidal P. 1992. HIMU-EM: The French Polynesian connection. Earth Planet. Sci. Lett., 110: 99-119.

Cornacchia M, Dars R. 1983. Un trait structural majeur du continent Africain. Les linéaments centre-africains du Cameroun au Golfe d'Aden. Bull. Soc. Géol. Fr., 25: 101-109.

Dautria JM, Girod M. 1986. Les enclaves de lherzolite à spinelle et plagioclase du volcan de Dibi (Adamaoua, Cameroun) : des témoins du manteau anormal. Bull. Minéral., 109: 275-286.

DePaolo DJ. 1981. Trace element and isotopic effects of combined wall-rock assimilation and fractional cristallization. Earth Planet. Sci. Lett., 53: 189-202.

Déruelle B. 1994. Le magmatisme de la "Ligne du Cameroun": le bilan positif des deux dernières décennies de coopération franco-camerounaise. Pangea, 30-36.

Déruelle B, Ngounouno I, Demaiffe D. 2007. The 'Cameroon Hot Line' (CHL): A unique example of active alkaline intraplate structure in both oceanic and continental lithospheres. $C$. $R$. Géoscience, 339: 589-600.

Dorbath C, Dorbath L, Fairhead JD, Stuart GW. 1986. A teleseismic delay time study across the Central African Shear Zone in the Adamawa region of Cameroon, West Africa. Geophys. J.R. Astr. Soc., 86: 751-766.

Dorbath L, Dorbath C, Stuart GW, Fairhead D. 1984. Structure de la croûte sous le plateau de l'Adamaoua (Cameroun). $C$. R. Acad. Sci., 298(12): 539-542.

Dumont J-F. 1987. Étude structurale des bordures nord et sud du plateau de l'Adamaoua: influence du contexte atlantique. Géodynamique, 2: 55-68.

Dupuy C, Barsczus HG, Liotard JM, Dostal J. 1988. Trace element evidence for the origin of oceanic islands basalts: an example from the Austral islands (French Polynesia). Contributions to Mineralogy and Petrology, 98: 293-302.

Ezangono J, Déruelle B, Ménard J-J. 1995. Benmoreites from Tchabal Djinga volcano (Adamaoua, Cameroon): products of kaersutite + plagioclase assimilation by a trachytic magma. Terra Abstr. Suppl. Terra Nova, 5: 1-161.

Fitton JG. 1987. The Cameroon line, West Africa: a comparison between oceanic and continental alkaline volcanism. In: 
Fitton JG \& Upton BCG (eds.), Alkaline Igneous Rocks. Geol. Soc. Spec. Publ. London, 30: 273-291.

Gèze B. 1943. Géographie physique et géologie du Cameroun occidental. Mém. Mus. Hist. Nat. Nouv. Sér., 17: 1-272.

Girod M, Dautria J-M, Ball E, Soba D. 1984. Estimation de la profondeur du Moho sous le massif volcanique de l'Adamaoua (Cameroun) à partir de l'étude d'enclaves de lherzolite. $C$. $R$. Acad. Sci., 298(16): 699-704.

Gouhier J, Nougier D. 1974. Contribution à l'étude volcanologique du Cameroun ("Ligne du Cameroun"-Adamaoua). Ann. Fac. Sci. Univ. Yaoundé, Yaoundé, Cameroun. 17: 3-48.

Green TH. 1994. Experimental studies of trace-element partitioning applicable to igneous petrogenesis - Sedona 16 years later. Chem. Geol., 117: 1-36.

Green TH, Edgar AD, Beasley P, Kiss E, Ware NG. 1974. Upper mantle source for some hawaiites, mugearites and benmoreites. Contrib. Mineral., 48: 3343.

Halliday A N, Dickin A P, Fallick A E and Fitton J G. 1988. Mantle dynamics: A $\mathrm{Nd}, \mathrm{Sr}, \mathrm{Pb}$ and $\mathrm{O}$ isotopic study of the Cameroon Line volcanic chain. $J$. Petrol., 29: 181-211.

Halliday AN, Davidson JP, Holden P, DeWolf C, Lee DC, Fitton JG. 1990. Traceelement fractionation in plumes and the origin of HIMU mantle beneath the Cameroon line. Nature, 347: 523-528.

Hart SR, Hauri EH, Oschmann LA \& Whitehead JA. 1992. Mantle plumes and entrainment-Isotopic evidence. Science, 256: 517-520.

Lasserre M. 1961. Contribution à l'étude géologique de l'Afrique. Étude de la partie orientale de l'Adamaoua (Cameroun Central). Bull. Dir. Min. Géol., 4(1): 1-31.

Leake BE, and 20 co-authors. 1997. Nomenclature of amphiboles: Report of the subcommittee on amphiboles of the international Mineralogical Association on new minerals and mineral names. Mineral. Mag., 61: 295-321.

Lee D-C, Halliday AN, Davies GR, Essente EJ, Fitton JG, Temdjim R. 1996. Melt enrichment of shallow depleted mantle: a detailed petrological, trace element and isotopic study of mantle-derived xenoliths and megacrysts from the Cameroon line. J. Petrol., 7: 15-441.

Marzoli A, Renne PR, Piccirillo EM, Francesca C, Bellieni G, Melfi AJ, Nyobe JB, N'ni J. 1999. Silicic magmas from the continental Cameroon Volcanic Line (Oku, Bambouto and Ngaoundere): ${ }^{40} \mathrm{Ar}-{ }^{39} \mathrm{Ar}$ dates, petrology, $\mathrm{Sr}-\mathrm{Nd}-\mathrm{O}$ isotopes and their petrogenetic significance. Contrib. Mineral. Petrol., 35: $133-150$.

McDonough WF, Sun SS. 1995. The composition of the Earth. Chemical Geology, 120: 223-253.

Moreau C. Regnoult J-M, Déruelle B, Robineau B. 1987. A new tectonic model for the Cameroon Line, Central Africa. Tectonophysics, 139: 317-334.

Morimoto N, Fabriès J, Ferguson AK, Ginzburg IV, Ross M, Seifert FA, Zussman J, Aoki K, Gottardi G. 1988. Nomenclature of pyroxenes. Mineral. Mag., 52: 535-550.

Ngangom E. 1983. Etude tectonique du fossé Crétacé de la Mbéré et du Djérem, SudAdamawa, Cameroun. Bull. Centr. Rech. Explor. Prod. Elf-Aquitaine, 7: 339-347.

Ngounouno I, Déruelle B, Demaiffe D. 2000. Petrology of the bimodal Cenozoic volcanism of the Kapsiki plateau (northernmost Cameroon, Central Africa). J. Volcanol. Geothermal Res., 102: 21-44.

Ngounouno I, Déruelle B, Demaiffe D, Montigny R. 2003. Petrology of the Cenozoic volcanism in the upper Benue valley, northern Cameroon (Central 
Africa). Contrib. Mineral. Petrol., 145: 87-106.

Ngounouno I, Déruelle B, Montigny R, Demaiffe, D. 2006. Les camptonites du mont Cameroun, Cameroun, Afrique. $C$. R. Geoscience, 338: 537-544.

Nkouandou OF. 2006. Pétrologie du volcanisme au nord et à l'est de Ngaoundéré. Thèse NR, Université Pierre et Marie Curie (Paris-6), Paris, p. 120.

Nkouandou OF, Ngounouno I, Déruelle B, Ohnenstette D, Montigny R. Demaiffe D. 2008. Petrology of the Mio-Pliocene Volcanism to the North and East of Ngaoundéré (Adamawa, Cameroon). C.R. Geoscience, 340: 28-37.

Nono A, Déruelle B, Demaiffe D, Kambou R. 1994. Tchabal Nganha volcano in Adamawa (Cameroon): petrology of a continental alkaline lava series. $J$. Volcanol. Geothermal Res., 60: 147-178.

Okereke CS. 1988. Contrasting modes of rifting: the Benue trough and the Cameroon volcanic line, West Africa. Tectonophysics, 7: 775-784.

Olafsson M, Eggler DH. 1983. Phase relations of amphibole, amphibole-carbonates, and phlogopite-carbonate peridotite; petrologic constraint on the asthenosphere. Earth and Planetary Science Letters, 64: 305-315.

Pouchou JL, Pichoir F. 1991. Quantitative analysis of homogeneous or stratified microvolumes applying the model «PAP». In Electron Probe Quantification, Heinriche DE, Newbury (eds). Plenum Press: New York; 31-75.

Poudjom Djomani YH, Diament M, Wilson M. 1997. Lithospheric structure across the Adamawa plateau (Cameroon) from gravity studies. Tectonophysics, 273: 317-327.

Poudjom Djomani YH, Nnange JM, Diament M, Ebinger CJ, Fairhead JD. 1995. Effective elastic thickness and crustal thickness variations in west- central Africa inferred from gravity data. J. Geophys. Res., 100: 22047-22070.

Shaw DM. 1970. Trace element fractionation during anatexis. Geochim. Cosmochim. Acta., 34: 237-243.

Stormer Jr J C. 1983. The effects of recalculation on estimates of temperature and oxygen fugacity from analyses of multicomponent iron-titanium oxides. Am. Mineral., 68: 586-594.

Stracke A, Hofmann AW, Hart SR. 2005. FOZO, HIMU, and the rest of the mantle zoo. Geochem. Geophys. Geosyst., 6(5): $1-20$.

Stuart GW, Fairhead JD, Dorbath L, Dorbath C. 1985. A seismic refraction study of the crustal structure associated with the Adamawa Plateau and Garoua Rift, Cameroon, West Africa. Geophys. J. R. Astr. Soc., 81: 1-12.

Tchameni R, Pouclet A, Penaye J, Ganwa AA, Toteu SF. 2006. Petrography and geochemistry of the Ngaoundéré PanAfrican granitoïds in central north Cameroon: Implications for their sources and geological setting. J. Afr. Earth Sci., 44: 511-529.

Temdjim R. 1986. Le volcanisme de la région de Ngaoundéré (Adamaoua Cameroun). Etude volcanologique et pétrologique. Thèse 3e cycle, Université ClermontFerrand II, Clermont-Ferrand, p. 180.

Temdjim R. 2005. Contribution à la connaissance du manteau supérieur du Cameroun au travers de l'étude des enclaves ultrabasiques et basiques remontées par les volcans de Youkou (Adamaoua) et de Nyos (Ligne du Cameroun). Thèse de Doctorat d'Etat, Université de Yaoundé1, Yaoundé, p. 339.

Temdjim R, Njilah IK, Kamgang P, Nkoumbou C. 2004. Données nouvelles sur les laves felsiques de Ngaoundéré (Adamaoua, ligne du Cameroun): chronologie $\mathrm{K}-\mathrm{Ar}$ et pétrologie. African Journal of Science and Technology 
(AJST), Science and Engineering, 5(2): 113-123.

Thornton CP, Tuttle OF. 1960. Chemistry of igneous rocks, I. Differentiation Index. Amer. J. Sci., 258: 664-684.

Toteu SF. 1990. Geochemical characterization of the main petrographical and structural units of Northen Cameroon: implications for Pan-African evolution. Journal of African Earth Sciences, 10: 615-624.

Villemant B, Treuil N. 1983. Comportement des éléments en trace et majeurs dans la série alcaline de Velay. Comparaison avec la chaîne des puys (Massif Central français). Bull. Minéral., 106: 465-486.
Weaver BL. 1990. Geochemistry of highlyundersaturated ocean island basalt suites from the South Atlantic Ocean: Fernando de Noronha and Trindade islands. Contrib. Mineral. Petrol., 105: 502-515.

Weaver BL. 1991. The origin of ocean island basalt end-member compositions: trace element and isotopic constraints. Earth Planet. Sci. Lett., 104: 381-397.

Zindler A, Hart S. 1986. Chemical geodynamics. Ann. Rev. Earth Planet. Sci., 14: 493-571. 